\title{
FACT: THE UTILITY OF AN INTEGRATION OF FUNCTIONAL ANALYTIC PSYCHOTHERAPY AND ACCEPTANCE AND COMMITMENT THERAPY TO ALLEVIATE HUMAN SUFFERING
}

\author{
GLENN M. CALLAGHAN \\ San Jose State University
}

BRIAN P. MARX

Temple University

\author{
JENNIFER A. GREGG \\ VA Palo Alto Health Care System
}

BARBARA S. KOHLENBERG
University of Nevada School of Medicine,
Reno, and VA Medical Center-Reno

\section{ELIZABETH GIFFORD \\ VA Palo Alto Health Care System}

Functional analytic psychotherapy $(F A P)$ and acceptance and commitment therapy $(A C T)$ are 2 contemporary behavioral therapies designed to address complex clinical problems. The 2 therapies are described, and areas of convergence and divergence are discussed. A new psychotherapy integrating the 2-functional-analytic acceptance and commitment therapy (FACT) - is defined. It is argued that FACT enhances the utility of FAP or $A C T$ alone by expanding the target of therapy to include both interpersonal and intrapersonal client behaviors. In addition, the authors posit that the

Glenn M. Callaghan, Department of Psychology, San Jose State University; Jennifer A. Gregg and Elizabeth Gifford, VA Palo Alto Health Care System; Brian P. Marx, Department of Psychology, Temple University; Barbara S. Kohlenberg, University of Nevada School of Medicine, Reno, and VA Medical Center-Reno.

Correspondence regarding this article should be addressed to Glenn M. Callaghan, PhD, One Washington Square, Department of Psychology, San Jose State University, San Jose, CA 95192. E-mail: psych@email.sjsu.edu intervention technologies used in FAP and ACT enhance one another. The authors also address the following topics: populations for whom FACT would be most beneficial, supervision and training issues, current empirical research on FACT, and FACT's applicability and generalization.

Traditionally, behavior therapies have been limited to the use of analytic principles that focus on overt or observable variables and highly specified clinical issues (Follette, Naugle, \& Callaghan, 1996). More recently, however, there has been a gradual movement to develop forms of psychotherapy based on a radical behavioral or functional analytic perspective. These therapies seek to understand the variables important to causing and controlling effective and problematic client behaviors in a broader context and integrate contemporary analyses of behavior into assessment and treatment (e.g., Haynes \& O'Brien, 2000). Radical behavioral or functional analyses include the role of contextual variables as well as cognitive and affective events in the assessment and treatment of psychopathology. The advantage of these newer approaches rests with their ability to address both interpersonal problems 
(i.e., relationship issues) and intrapersonal difficulties (i.e., problems with experiencing emotions).

Over a decade ago, two newer behaviorally based interventions, acceptance and commitment therapy (ACT; Hayes, Strosahl, \& Wilson, 2000) and functional analytic psychotherapy (FAP; Kohlenberg \& Tsai, 1991), were outlined by the developers of each treatment (Kohlenberg, Hayes, \& Tsai, 1993). These treatments use a behavior analytic and contextual framework to approach complex clinical issues, including the therapeutic relationship and emotional acceptance. In other words, both FAP and ACT utilize basic behavioral principles, such as negative and positive reinforcement, escape, and avoidance and apply them to thoughts, feelings, and interpersonal interactions. At the level of theory or principle, these treatments do not differ from traditional behavior analytic or behavior therapy approaches; however, the focus of contemporary behavioral treatments is on less easily observable subject matter, such as thoughts and feelings.

Although each of these therapies was developed independently from one another, both treatments share this behavior analytic background, and research has been underway to demonstrate the effectiveness and utility of each approach. This article offers the next iteration in the development of these treatments, stipulating that both therapies have complementary and differing strategies for treating psychological phenomena. We argue here that both ACT and FAP can be used as part of the same assessment and treatment approach for clinical problems (Callaghan \& Gummeson, 2001; Paul, Marx, \& Orsillo, 1999).

This article presents a discussion concerning the principles of both therapies and the unique contribution each provides to behavioral psychology. Although each therapy by itself adds a great deal to the understanding and treatment of psychopathology, an argument can be made that neither treatment is comprehensive in its approach. The discussion hereafter focuses on the integration of the FAP and ACT approaches. This hybrid, termed FACT (an abbreviated acronym formed by simply adding the two separate terms together; Kohlenberg \& Gifford, 1998), creates a broader and more comprehensive therapy while still remaining theoretically consistent and true to the goals of each individual therapy. We suggest that this combined approach allows therapists to handle a variety of clinical problems that meet criteria for a formal diagnosis as well as those that fall outside the purview of identified problems using the Diagnostic and Statistical Manual of Mental Disorders (DSM; American Psychiatric Association, 2000). Before discussing the integrated treatment, we provide a brief overview of both therapies. Advantages and disadvantages of each therapy conducted by itself are described as well.

\section{ACT}

ACT is based on an analysis of language from a functional contextual perspective (Hayes, 1987, 1994; Hayes et al., 2000; Hayes \& Wilson, 1994). Put simply, this analysis suggests that it is common for humans to regard some of their own private reactions (e.g., physiological sensations, affect, cognitive evaluations, and perceptions) as aversive and to thus make attempts to modify or eliminate these reactions. In general, however, attempts to control such aversive private events tend to be ineffective and paradoxically result in more of the same thoughts and emotions that the individual was trying to avoid in the first place. In many instances, the individual may manage to achieve some short-term relief from these noxious thoughts, feelings, or sensations; however, this short-term relief often is associated with additional long-term difficulties. For example, the individual who drinks to control social anxiety may also develop a dependence on alcohol that might lead to other impairments (e.g., losing a job, being arrested for driving under the influence). Parents who fail to set limits with their child in order to reduce fears of parental incompetence may inadvertently help to foster severe child behavior problems. As a result of these continued difficulties, the individuals in both of these examples will undoubtedly experience more of the aversive private reactions that were to be avoided in the first place (negative social evaluation, fears of parental inadequacy, etc.).

ACT emphasizes acceptance as an essential skill that aids in moving clients toward their specified values. In this treatment, acceptance refers to the conscious abandonment of a mental and emotional change agenda (when change efforts do not work) and an openness to one's own emotions and the experience of others (Hayes, 1994). More specifically, the goals of ACT are to help the client recognize the ineffectiveness of experiential avoidance and to develop a new, 
more effective repertoire for experiencing painful thoughts and feelings. This new repertoire is always based on the client's personal set of values and goals for therapy.

Five therapeutic phases are implemented to accomplish these goals. In the first phase, clients are brought into contact with the reality that previous struggles to control their inner experiences have been unsuccessful. In the second phase, clients are helped to see that not only have their previous struggles to control private events been unsuccessful but that these struggles have actually made matters worse. The third phase of ACT emphasizes attempts to help clients delineate between their personal self and their cognitive, emotional, and physiological experiences. In the fourth phase, clients are asked to willingly experience the aversive private events that they have previously avoided to accomplish their goals not yet reached. Finally, the fifth phase of ACT involves securing a commitment from the client and implementing behavior change strategies.

Several studies have shown that ACT is efficacious when applied to affective disorders (Zettle \& Hayes, 1987; Zettle \& Rains, 1989); anxiety disorders, including obsessive-compulsive disorder and agoraphobia (Hayes, 1987); the emotional distress of families with severely physically handicapped children (Biglan, Glasgow, \& Singer, 1990); and preliminary studies on the treatment of substance abuse (Hayes et al., 2003). Additionally, in a randomized, controlled trial, Bach and Hayes (2002) found that psychotic patients receiving ACT had significantly lower rates of rehospitalization over a 4-month period.

Although there are multiple studies reporting the effectiveness of ACT with a number of populations, there are limitations to this approach to treatment. One key limitation results from the fact that problems related to emotional avoidance often involve others in the client's life. For example, if a client has difficulty experiencing sadness, this may have resulted from an interpersonal relationship in which the expression of that emotion was prohibited or even punished. When clients learn to experience rather than escape or avoid emotional experiences, they must, in turn, develop the skill of expressing those emotions with others. ACT can achieve a very important goal of helping clients to experience a feeling and not engage in strategies to avoid that feeling, but unless the client is skilled in talking with others about those feelings or the needs they have associated with those feelings, the client will have only a partially developed repertoire necessary for interpersonal interactions.

As an example, consider a female client who experiences social anxiety in situations in which she is asked to interact with people she does not know. In this example, this client is coming to treatment because she is disturbed by her fears of being evaluated by others. When she feels evaluated, she responds by limiting the extent to which she expresses herself to others or physically withdraws from all social situations. From an ACT perspective, work with this client would focus on helping her learn that attempts to reduce or change these thoughts and feelings related to social evaluation may only make her situation worse. ACT would also focus on helping her define what she values doing in these situationswhether she wishes to be somebody who shies away from relationships or somebody who engages them. If she values engaging relationships, the client would be assisted to become an observer of her thoughts and feelings in order to experience them and not leave the conversation or the room when they occur. The goal of therapy might be to help her experience her anxiety in order to help her "live" her value of engaging in relationships.

\section{FAP}

FAP (Kohlenberg, Hayes, \& Tsai, 1993; Kohlenberg \& Tsai, 1991, 1995; see also Callaghan, Naugle, \& Follette, 1996) operates from the assumption that much of psychopathology or human suffering is interpersonal in nature, and the therapeutic relationship is essential in bringing about clinical improvement. Although other behavioral writers have acknowledged the importance of the client-therapist relationship (e.g., Rosenfarb, 1992; Schaap, Bennun, Schindler, \& Hoodguin, 1993; Sweet, 1984), FAP has approached this analysis consistent with the principles of radical behaviorism and has specified the therapist behaviors required to bring about client change. One key assumption in FAP is that most client problems occur in the context of, or as a result of, interpersonal relationships. FAP also holds that client problems that occur outside of therapy with others will also occur in-session with the therapist. Given these assumptions, the therapist then has the unique opportunity to uti- 
lize the therapeutic relationship to help the client build more effective interpersonal skills by responding to those client behaviors that occur during therapy and by helping the client develop new responses to the therapist (and, consequently, other people). FAP is similar to other interpersonally oriented therapies in that it seeks to understand psychopathology as a function of interactions with people. It departs from these approaches by directing the therapist to respond to the client's behavior as it impacts the clinician in the moment.

The proposed mechanism of clinical change in FAP is the therapist's in-session contingent responding to client behavior as it occurs (see Follette et al., 1996). Targeted client behaviors, termed clinically relevant behaviors (CRBs), are determined from a conceptualization of the client's reported problems and goals for treatments (see Kohlenberg \& Tsai, 1991, for a more thorough discussion). The therapist's task in FAP is to elicit and then contingently respond to a client's problem behaviors (termed CRB1s) and more adaptive behaviors (termed $C R B 2 s$ ), as they occur in the therapy session. The therapist differentially reinforces and increases the rate of CRB2s while attempting to decrease the frequency of CRB1s. When it is appropriate, the therapist models and reinforces descriptions of the controlling variables for $\mathrm{CRB} 1 \mathrm{~s}$ and $\mathrm{CRB} 2 \mathrm{~s}$ (termed CRB3s).

To effectively reinforce client improvements and respond to problem behaviors, the therapist must establish him or herself as a trusted and liked individual. In other words, the therapist must become an effective and salient provider of social consequences, such as praise or critical feedback. The therapist uses this developing relationship with the client to help him or her engage with more effective skills in-session. The therapist then makes every effort to ensure that the client attempts these new behaviors outside of the session so that the client's improved repertoire generalizes to other settings. By and large, the therapist responds to the client as a representative of the client's social community. For example, if the client engages in a behavior that is ineffective with the therapist or would be ineffective in other interpersonal interactions (given the client's goals for these types of relationships), the therapist responds to the aversive or ineffective qualities of the client's behavior and helps him or her develop more prosocial and effective responses.

To accomplish the stated goals of FAP, the therapist must be vigilant for the occurrence of in-session CRB1s and CRB2s. The therapist works to elicit CRB1s and foster the development and expression of CRB2s during the therapy session. It is critical that FAP therapists acknowledge the expression of CRB2s in therapy with "natural" reinforcers rather than "contrived" reinforcers (or consequences) that clients would not experience outside of therapy (e.g., giving a token reinforcer to a client who has difficulties with anger management for demonstrating restraint). Using natural reinforcement for behavior increases the likelihood of the generalization and maintenance of more adaptive behavior outside of the therapy session. For FAP to be successful, the therapist must continually assess whether or not differential reinforcement has impacted the occurrence of targeted in-session problems and improvements. The critical outcomes for FAP are the alleviation of distress, the demonstration of improved functioning by clients both inside and outside of therapy, and the generalization of the client's in-session behavior to other external circumstances.

If we consider the socially anxious female client from an FAP perspective, the therapist will first need to identify what specific responses the client has in social situations and when these are more likely to occur. For example, perhaps the client can maintain a conversation that she has anticipated and keeps at a superficial level but becomes very anxious if the discussion strays from what was "scripted" in advance. The therapist determines the client's goals for treatment and uses these to guide the intervention. Let us assume that the client wants to be less socially anxious and to become more interpersonally connected to others. The FAP therapist then needs to watch for opportunities in the therapy session when the client begins to feel uncomfortable in the course of a discussion. If the client begins to withdraw from the therapist or becomes rigid with the course of the conversation, then the therapist will likely use this as an in-session opportunity to respond to the client's problem behavior. The FAP therapist will tell the client how this withdrawal, for example, affects him or her. The therapist may say, "Now, you look like something just changed. You've withdrawn from me as we were talking. That makes it very hard to 
be connected to you." This is a therapist's response to a CRB1, the client's problem behavior of social withdrawal. A general rule for FAP is to attempt to have the client engage in a more effective response in that moment, that is, to elicit a CRB2. The therapist might continue, "What else could you do? Right now, what could you try to do that would have you feel less disconnected?" If the client responds with an approximation toward this goal, the therapist relates how that feels, "This is really helpful, you're telling me you feel anxious, that it's hard for you to talk. Now I feel like we are working together on this problem. I feel connected to you again, and that's really the goal here."

One issue to consider when looking at examples of FAP responses is that they are often very idiosyncratic with respect to the therapist's style. Therapists may respond differently to the above situation in terms of how they give feedback to problem behaviors and how they attempt to reinforce a CRB2. An essential feature of FAP is that the responses can vary and that the therapist is always looking at the function of the response, that is, what the therapist's response actually did. If a therapist responds to a client's improved response in-session by saying, "OK, great, you are making progress" but this does not serve to increase the likelihood of the client making that same improvement, it is not an effective therapist response. This freedom from proscriptive rules about style can be frustrating to novice therapists looking for explicit instructions about what to say in therapy. However, the ability for the therapist to use his or her style in the room, while watching to see how it actually affects the client, allows for much more genuine and effective responding. If a therapist responds disingenuously, the client will likely experience this, and the response will have little impact on the client's behavior in or out of session. The key to FAP is that the therapist attempt to provide consequences to the client's behavior. When the client's responding works well to accomplish his or her goals, the therapist provides social reinforcers (praise, commenting on becoming closer, understanding the client). When the client's responses are less effective, the therapist lets the client know how that impacts him or her (feeling distant, not being able to be helpful to the client).

Research is still being conducted with FAP to determine its effectiveness in treating client problems (Gaynor, 2002; Kohlenberg et al., 2004).
Several recent larger scale studies suggest that FAP may be an effective treatment for adolescents (Gaynor \& Lawrence, 2002) and for depression (Kohlenberg, Kanter, Bolling, Parker, \& Tsai, 2002). In addition, open trials are being conducted to determine the effectiveness of FAP with anxiolytic dependence and nicotine dependence. A handful of single-subject research studies have been conducted indicating promising results for the treatment of personality disorders (Callaghan, Summers, \& Weidman, 2003) and an adjunctive treatment for posttraumatic stress disorder (Prins \& Callaghan, 2002).

One potential problem with FAP is that, in an effort to engender more effective interpersonal behaviors, FAP therapists may inadvertently shape and reinforce experiential avoidance behaviors, an outcome contraindicated by ACT. For example, consider a male client who has difficulty with interpersonal conflict. During a particularly hard session, he feels angry toward his therapist and states that he wishes to change the topic of discussion. Because the CRB1 has been identified as a difficulty with conflict or the unwillingness to be assertive in session and because the improvement (CRB2) has been defined as increases in assertive behaviors, the FAP therapist may then respond to the client by honoring his request to talk about something else. Although the therapist would indeed be reinforcing an instance of assertive behavior, the difficulty is that the therapist may also be helping the client escape those feelings of anger. Provided that the therapist changes to accommodate the client, this client now has learned the means by which he can escape from difficult affective experiences.

\section{Commonalities and Differences}

Clearly, there are strengths and weaknesses to each of the two treatment approaches. Both FAP and ACT are based on behavioral principles; however, each brings a different focus to the types of problems that can be addressed in the treatment. Highlighting commonalities and differences allows a better understanding of the challenges of simply having one therapy supercede the other (see discussion that follows) and points to the need for an integration of the two treatments.

\section{Commonalities of ACT and FAP}

FAP and ACT assume that behaviors are best understood through an operant analysis of behav- 
iors under the control of particular stimuli and maintained by particular consequences. In other words, both of these treatments have a behavioral conceptualization as their foundation, and it is from this position that problems are addressed and improvements measured. Consistent with this conceptualization of psychopathology, the improvements sought by both treatments are healthier client repertoires that help the individual function more effectively in other situations given their values and goals for living. While insight into the client's problems is not a specific goal for therapy, both FAP and ACT attempt to help clients determine what is effective and ineffective in terms of their own behavior in order to help them recognize conditions when ineffective behavior is likely to occur and when they may be able to be more effective in achieving their goals. This awareness is not necessary for behavior change to occur for either treatment, but it may be useful under some circumstances. From an FAP and ACT perspective, a client could become more effective experiencing his or her feelings or interacting with others without necessarily having insight or awareness about all of the variables that make this happen. A client may, however, find that it is useful to be aware of when difficulties are more likely to occur and when he or she should, for example, attempt to practice what was learned in therapy. The following point relates this to the case of the socially anxious client, from both an ACT and FAP perspective: While the factors the client considers responsible for her social anxiety would be discussed, the amelioration of her distress would not be presumed to occur through discussion of the cause of the client's anxiety. Rather, her identification of situations in which she is likely to feel anxious would be used to talk about the different responses she could make that may be more or less helpful to her accomplishing her goals.

Another key commonality lies in the requirement that clients try new strategies for accomplishing their goals when the previous responses no longer work. Both ACT and FAP therapists help the client recognize when these older behavior patterns are not working toward meeting the client's goals and prompt the client to attempt new behaviors. These therapies value and promote this generativity and creativity in response to ineffective behaviors so that opportunities for more effective responding may occur.
Perhaps the most notable commonality of ACT and FAP is the emphasis on process. Although the content of the client's struggles with different life events remains important, in both therapies there is an emphasis on what the client does in response to those struggles-especially what the client does in the session. For example, a client may come into session after a difficult event and talk about everyday content. Depending on the case conceptualization, this process-and particularly the impact that this process has-would likely be discussed in both ACT and FAP. In the case of the socially anxious client, this element may be most easily identified in situations in which the client exhibits socially anxious responding with the therapist. From either an ACT or FAP perspective, this type of responding would be targeted and explored directly in the therapy session.

Finally, both ACT and FAP are very compassionate therapies. Both treatments approach human suffering with profound empathy for how difficult a client's problems are for that person. Each therapy assumes that the client is doing exactly what his or her own personal learning history would produce. Therapists conducting either treatment assume that the client's behavior would be different only if the client had a different history than he or she has had. This focus on the importance of an evolving history in producing current behavior can provide therapists and clients with the hope that developing an additional, newer learning history may allow the client to engage in more effective behaviors. These behaviors may then build skills that allow for more success in reaching the client's personal goals. For these reasons, both FAP and ACT therapists are extremely empathetic and optimistic about the possibility for behavior change.

In the case of the socially anxious client, there are many ways in which this empathy would likely be conveyed by either an ACT or FAP therapist. First, the distress related to having unwanted anxiety would be validated in either treatment, and the difficulty of having so much anxiety in the client's life would be discussed. Additionally, from both an ACT and FAP perspective, empathy for what the client is missing by avoiding social interactions would be discussed, both to validate what the client is missing and to serve as a motivator for behavior change. 
Integration of FAP and ACT

\section{Differences Between ACT and FAP}

The primary distinction between ACT and FAP is the specific emphasis on intra- versus interpersonal behavioral problems, respectively. ACT focuses specifically on the emotional experience of the client with less consideration of the interpersonal context in which the feeling occurs. FAP emphasizes the importance of how the client expresses his or her feelings or values sometimes to the detriment of not knowing if the client is capable of even having these feelings or knowing what his or her values are. This is not a criticism of the therapies as inadequate. Each was developed to address different issues. However, these differences do create challenges for a complete conceptualization and treatment of client problems. The disparities also create difficulties for therapists attempting to supplement each treatment by adding what appears to be absent.

The first difference between the two treatments lies in the evaluation of and provision of consequences to ineffective client behaviors that occur during the session. FAP assumes that therapeutic improvements occur as the therapist naturally reinforces more adaptive, healthier behavior by the client. A FAP therapist pays particular attention to the power of consequences in the therapy relationship. Thus, the reactions that the therapist has to the client's behavior are critical. The therapist does not judge or negatively evaluate the client, as a person, when his or her behavior is not effective, but he or she will evaluate and provide consequences to the behavior. For example, if a socially anxious client ignores a FAP therapist's praise, the therapist might say, "When you ignored my supportive statements about you effectively handling that situation, I felt hurt and angry, and I wanted to shut down for a moment." In general, the FAP therapist uses the therapeutic relationship to create behavior change by reacting to and commenting on the impact that client behavior has on him or her.

In contrast, an explicit evaluation of a client's thought or other experience as it is expressed insession is typically contraindicated in ACT as it is believed that this will serve to strengthen the client's own conception that evaluation is inherently important or meaningful. In contrast to the evaluative process of FAP, ACT therapists seek to (a) facilitate clients' awareness of their own thoughts and feelings, including their own evalu- ations, (b) encourage clients to consider thoughts, feelings, sensations, and memories as nothing more than thoughts, feelings, sensations, and memories, and (c) understand that if the client should choose to act on the basis of his or her thoughts or feelings, it should be done in accordance with the standards set by the client's values and not according to any evaluations or standards established by the therapist or anyone else. In the example of the socially anxious client ignoring praise by the therapist, an ACT therapist might explore with the client what she was experiencing when the therapist praised her and might look for opportunities for her to experience any anxiety she might have felt without trying to avoid it. Also, the therapist might explore with the client whether ignoring the therapist's praise is what she would value doing in such a situation.

\section{FACT: Integration and Utilization}

Despite the fact that FAP and ACT posit different therapeutic change agents, these systems are not antithetical or contrary. In fact, FAP and ACT are complimentary therapy systems. Both of these therapies are based on a behavioral conceptualization of psychopathology, emphasize the development of new behavioral repertoires, and rely on the therapeutic process to evoke behavior change. Most important, both systems assume that many client problem behaviors are under the control of aversive stimuli and consist of escape and avoidance behaviors. Given the complimentary nature of the two systems, it seems reasonable to believe that a combination of both systems may serve to improve therapeutic effectiveness.

\section{Different Approaches to Integration}

There are at least three different approaches to integrating FAP and ACT into one treatment system: (a) utilizing the therapeutic relationship within ACT (see, e.g., Blackledge \& Hayes, 2001); (b) utilizing acceptance repertoires within FAP (see, e.g., Cordova \& Kohlenberg, 1994); and (c) fully integrating ACT and FAP. Of these three approaches, we posit that a balanced synthesis of ACT and FAP, where each therapy provides the context for doing the other, is a much more comprehensive system of psychotherapy and may provide an opportunity for optimal behavioral change. 
The problem with the first approach is that the therapist can no longer respond to the aversive social impact that the client has on the therapist. The ACT therapist in this case risks developing a client's repertoire that is effective in therapy but that creates problems for the client in other social situations. Although the ACT therapist focuses on replacing the client's escape and avoidance strategies, he or she may not help develop the client's interpersonal skills sufficiently to interact with others effectively.

One challenge of the second approach, of subsuming ACT under FAP, lies with the inconsistencies of therapist responding that will occur over the course of therapy as the therapist tries to do both as distinct interventions. More specifically, when the client engages in a behavior that aversively impacts the therapist because of the way in which the client is attempting to avoid his or her feelings, the FAP therapist is required to talk about how this avoidance affects him or her, whereas the ACT therapist helps the client learn to experience those feelings as they occur. Without properly integrating the two treatments, the therapist would be conflicted about which strategy to use with the client.

\section{An Integration of ACT and FAP}

Allowing both ACT and FAP to become the context for each other solves the quandary of deciding which therapy should serve as the foundation of the other and provides a more comprehensive approach. In our formulation, each therapy is done simultaneously. The premise for FACT is that most humans are fundamentally social beings and that much of what we do is a consequence of social reinforcers. Moreover, humans are also verbal beings, and much of human suffering is brought about by the unique functions of language (e.g., Hayes, Gifford, \& Wilson, 1996). ${ }^{1}$ FACT capitalizes on the strengths of FAP and ACT by focusing on the difficulties that emerge from experiential avoidance repertoires and shaping experiential acceptance skills in the service of client values while using the contingent reinforcement available in-session, between the client and the therapist.

It is difficult to imagine that the process of altering interpersonal repertoires (conducting FAP) does not require a client's willingness to experience aversive emotional experiences. Likewise, it is equally challenging to suggest that ex- periential avoidance and the strategies employed to engage in acceptance (doing ACT) do not occur in the context of interpersonal relationships. Each therapy requires the assumptions of the other to capture the complexities of psychological phenomena.

For example, to work on creating new interpersonally effective repertoires, a therapist must clarify that the client values intimate interpersonal relationships. If so, then the therapist can use the therapeutic relationship to develop new interpersonal strategies. Therapists should recognize, however, that trying out new behavioral repertoires may be stressful and difficult for clients, and they may experience aversive thoughts and feelings associated with their respective successes and failures. As a result, success in such endeavors will be more likely if clients have already developed an ability to endure or accept these thoughts and feelings, do not allow such private experiences to impede progress, and persist in trying new ways to relate interpersonally. In other words, FAP requires ACT: Achieving new interpersonal repertoires necessitates an ability to experience aversive affective states.

In much the same way, ACT requires FAP. No psychological experience occurs in a vacuum. Most, if not all, of our thoughts and feelings occur in the context of other people, even if those people are not physically present. Although most human beings value and desire close relationships with others, this desire is often counterbalanced by a fear of vulnerability and reluctance to develop close relationships. This conflict leaves humans, and clients in particular, in a difficult position. The therapeutic relationship can be utilized to give the client a place to attempt the behaviors that bring about interpersonal closeness in a safe place. Indeed, it is in the context of the therapeutic relationship that any ACT therapy occurs; utilizing this rich opportunity to enhance skill development is a logical choice. When the client engages in improved behaviors in-session, the therapist can reinforce the efforts on the basis of the impact that they have on the therapist and specifically how close the therapist feels to the client when this repertoire is engaged. This more context-specific reinforcer may serve to promote

\footnotetext{
${ }^{1}$ We recognize that not all humans have verbal capabilities. We are attempting to characterize those humans with language capacity that receive psychotherapy services.
} 
the client's continued attempts in treatment and allow the client to try these strategies in other settings.

Returning to the example of the socially anxious client, from an integrative FACT perspective, the treatment would simultaneously target both intra- and interpersonal targets. Assuming that the client valued close interpersonal relationships, the therapeutic relationship would be the place in which the client was given an opportunity to experience anxiety-provoking interpersonal interactions, in order to notice this anxiety as only that feeling and remain in the experience with the therapist. At the same time, the therapist would provide information about how the client's behavior impacted the closeness the therapist detected between them in order to develop the client's ability to connect successfully with others outside of therapy. For example, the therapist may see the client withdraw and respond to that withdrawal similarly to the in-session problem behavior as with the previous FAP example, "You look like you are really anxious, and you are changing the topic now. This makes it hard for me to know what to do, to know what you want that would help." From a FACT perspective, the therapist would then ask the client to notice the experience of anxiety for what it is, an experience, and to see the emotional avoidance as both a problem for the client on his or her own and one that impacts the client's interpersonal closeness with others. The therapist might say, "Can you have this anxiety right now, for what it is, and stay with it? Could we keep talking about what we are discussing, and have you feel anxious?" If the client is able to do this, the FACT therapist will reinforce the client improvement in-session and will have altered both the client's interpersonal skill set as well as his or her interpersonal ability to accept emotional experiences.

\section{Practicing FACT}

\section{Populations Appropriate for FACT}

As with many treatments rooted in behavioral principles, the type of population appropriate for FACT is not nearly as important as the defined and targeted problems. In other words, FACT is not for the treatment of topographically defined syndromes such as posttraumatic stress disorder or major depressive disorder; it is designed to treat the problems specific to those individuals who may or may not meet criteria for those syndromes. If a client has difficulty with interpersonal relating, experiencing aversive emotions, or avoiding specific interactions as a result of a traumatic event, then those behaviors are targeted and worked on in therapy. That said, the FACT therapist is less concerned with identifying and diagnosing disorders than specifying the problems that occur with respect to experiential avoidance or interpersonal closeness.

With regard to the syndromes presented by the $D S M$, in principle, FACT may be useful for any behavioral disorder, whether it is formally diagnosed or fails to meet formal criteria (e.g., personality disorder not otherwise specified; Callaghan et al., 2003). The advantage of using FACT is that a broad array of issues, from those primarily focusing on interpersonal difficulties to those involving faulty avoidance repertoires, can be addressed. In other words, this synthesized treatment can be used for those clients with problems that appear private in nature and cannot be addressed only with an interpersonally focused treatment like FAP. It also can be used for those clients with problems of broad interpersonal repertoire deficits that cannot be treated only with an acceptance-based treatment like ACT.

It is recommended here that therapists utilize empirically supported treatments (ESTs) for heavily researched problems like panic disorder or major depressive disorder. The supplementary use of FACT in these cases may further enhance the effectiveness of well-established ESTs. FACT would certainly be helpful in cases in which a client fails to meet criteria for one of these ESTs or is nonresponsive to a typical course of treatment.

The FACT approach may be especially valuable for treating long-standing, pervasive repertoire problems as found among the Axis II personality disorders of the DSM. Other longstanding difficulties that are similar to these characterological deficits found on Axis I include dysthymia, generalized anxiety disorder, and cyclothymia. These disorders involve maladaptive interpersonal behaviors as well as an inability to tolerate and a desire to escape from aversive psychological experiences. With one notable exception (see Linehan, 1993), currently no ESTs exist for Axis II problems and few therapies have been developed for the more pervasive Axis I disorders despite their high comorbidity with other diagnosed disorders. 
Examples of Clinical Assessment and Treatment Using FACT

The primary assumption of FACT is that therapy requires an assessment and treatment of both interpersonal problems and problems the client has with experiential avoidance. FACT therapists assume that these problems occur both in and out of the therapy session. Thus, a FACT therapist may address (a) the difficulty of feeling what there is to be felt and (b) the difficulty in expressing these feelings to other individuals. When a client presents for therapy, the FACT therapist may assess the client's values and goals for treatment. The therapist then may assess the client's behavior problems and delineate targeted responses for treatment (see, e.g., Hawkins, 1986). With respect to the first issue, each client comes to therapy with his or her own personal learning history. That the client has sought treatment suggests that he or she recognizes that some aspect of his or her life is not going well, and he or she does not know what to do (though the client may not yet realize that his or her attempts to minimize pain are ineffective). In FACT, assessment entails determining whether the client utilizes maladaptive avoidance strategies, determining what these strategies may be, and evaluating the client's interpersonal relationship skills.

Assessment is conducted in several ways. First, the therapist can ask the client directly what the specific problems are that he or she is having. The therapist will then attempt to specify these problems as targets for intervention. This includes determining when, where, how often, and the strength of each of the responses. These problem behaviors of the client are then assessed as interpersonal and intrapersonal issues. For example, if the client appears to be sad or depressed, the therapist will specify what the client is doing that may be maintaining this sadness or preventing the client from experiencing other feelings. A depressed client may be doing a variety of things, including attempting to avoid feelings of sadness, and he or she may be unable to effectively interact with others to get support in a difficult time. This FACT assessment must include a clarification of the client's values and barriers (both interpersonally and intrapersonally) to living a life consistent with those values. This values assessment is then directly translated into specific goals for therapy.

A second form of FACT assessment is con- ducted in vivo. As the client and therapist interact early in therapy, the therapist is watching to see how the client engages both his or her own emotional experience and the therapeutic relationship. In this case, the therapist is looking for instances in which the client avoids or attempts to control his or her feelings in-session, particularly in a way that interpersonally disconnects the client from the therapist. With a depressed client, the therapist will be looking for examples of the client withdrawing or expressing disingenuous emotions, such as putting on a false, brave front. During the assessment phase of FACT, the therapist will comment on these responses as they happen to inquire about whether and when these behaviors occur outside of therapy. This lets the therapist know the pervasiveness of the problem.

Following assessment, the therapist determines how to observe or evoke these behaviors insession, to naturally respond to them when they are ineffective, and then shape a more effective set of skills. A key to clients developing new behaviors is a therapeutic environment that provides them with a safe context to experience previously avoided thoughts and feelings. During treatment, when addressing targeted client problems, the therapist shapes the client's ability to disclose aversive emotional experiences. Sharing such experiences with the therapist also serves to enhance interpersonal intimacy with the therapist. As the client struggles with experiencing thoughts and feelings evoked by the process of trying new ways to relate more effectively and as the therapist provides feedback that the old ways of relating are not effective for the client, the client may realize that therapy is more of a struggle than previously realized. However, the additional realization that the therapist appreciates the client's willingness to struggle with the experience may provide the client with the proper supportive context needed to continue in therapy.

When the client is required to experience something he or she is frequently unwilling to feel or is given feedback that what he or she just said did not work to bring the therapist closer to the client or to facilitate change, the client may want to escape from the experience or even leave treatment altogether. Examining and then reminding clients about the cost of not experiencing the painful thoughts and feelings that arise allows the treatment to continue. These skills are designed to then generalize to other interpersonal interactions for the client outside of therapy. Cli- 
ents are reminded that they are working toward living their value of having effective relationships with others, not just with their therapist. However, the relationship with the therapist is a place to learn these skills.

Holding a behavioral perspective. It should be made explicit to the reader that the therapeutic interventions presented here stem from a very specific paradigm, that of learning theory. Some of the interventions described and illustrated, therefore, may not be obvious to readers as necessary or effective. Still, all of these interventions remain consistent with the paradigm. If a therapist were to adopt a behavioral perspective, it would make it easier to understand some of the interventions. Indeed, it would be very helpful for a therapist to have had practice conducting behavior analyses focusing on what each client response is accomplishing for him or her (e.g., preventing them from having to feel aversive feelings) and to attempt to determine what comes before and after that behavior that maintains the response. These are activities required by one who takes a behavioral perspective to treatment and would likely make the interventions described here more easily adopted. That said, some practitioners of both ACT and FAP argue that it is not necessary to adopt a behavioral perspective to conduct these interventions; there are easily understood techniques within the therapies that can be done by all therapists from all theoretical orientations. Stated more plainly, from an ACT therapist's perspective, it would benefit many interventions to focus on a client's ability to experience thoughts and feelings rather than attempt to eliminate them. From a FAP approach, most therapies would benefit from examining what is going on in the therapy session, between the client and therapist, and to utilize in-session examples of interpersonal problems as opportunities to help the client change. We ask the reader to determine what technologies or specific treatment strategies are extractable from this brief discussion of the two interventions. If readers see the utility of either or both approaches, we suggest continued reading with some of the references provided in the text.

FACT in supervision and training. There is a distinct advantage in using the principles of FACT to train beginning therapists, as they often experience concerns about competence or performance and are anxious about being able to effectively help clients. Training in FACT allows the therapist to develop an acceptance repertoire for his or her own experiences, particularly those of anxiety around conducting effective psychotherapy. For instance, trainees are often concerned with evoking and responding to client problem behaviors (CRB1s) in-session, and novice therapists frequently become anxious about responding effectively and not in a way that is interpreted as excessively aversive by the client. If a therapist does respond and attempts to evoke a CRB2 and the client still engages in a problem response, the therapist's anxiety may increase even more. This may work to set up an aversive feedback loop for the therapist, who may become increasingly self-evaluative and anxious. This almost invariably disrupts the session or at least the therapist's ability to respond effectively. At any point in this cycle, the therapist may focus on escaping from his or her own aversive experiences, which in turn would compromise the treatment the client receives. In FACT, therapists-intraining are helped to engage their own acceptance repertoire so that they are not caught in this difficult loop and so that they stay focused on the goals of therapy.

Clinical research on FACT. There are already examples in the empirical literature and projects underway that illustrate efforts to combine ACT and FAP. For example, Paul et al. (1999) treated a court-referred exhibitionist using a combination of both ACT and FAP techniques. This treatment focused on the client's acceptance of previously intolerable affective states as well as social anxiety, exhibitionism, and use of marijuana. The client's urges to expose, acts of exhibitionism, and drug use were assessed during 12 months of treatment and at a 6-month follow-up. Results indicated that the client's urges to expose and frequency of public masturbation at treatment termination and at follow-up assessment were significantly reduced from baseline. Further, the treatment facilitated social-skills development and reduced drug use as well as symptoms of depression and anxiety. The results of the combined intervention suggest that FACT can be successfully applied to treat sexual deviance and other problems.

Gifford, Kohlenberg, and colleagues are currently conducting treatment development research utilizing FACT. This research project is focused on developing and evaluating a FACT approach to smoking cessation. The rationale for the use of FACT for smoking cessation is based 
in part on the link between nicotine use and negative emotional states as related to smoking relapse (e.g., Cinciripini, Hecht, Henningfield, Manley, \& Kramer, 1997; see Piasecki, 2000). In addition, the use of social support in smokingcessation treatments is one of the validated elements in the AHCRP guidelines for efficacious smoking-cessation treatments (Fiore et al., 1996). This research project is investigating whether FACT, a treatment that explicitly combines the teaching of acceptance skills in the context of a therapeutic relationship, is an effective approach to providing contingent reinforcement for sustaining contact with previously avoided emotional experience in an effort to reduce or eliminate smoking tobacco. The project is ongoing, and the data have not yet been analyzed.

The challenge of generalization. All psychotherapy is faced with the question of whether behaviors learned during the treatment session will generalize to the client's life outside of the therapy hour. The onus rests on the providers of treatment to ensure that this has occurred. ACT addresses this by emphasizing the client's ability to engage in an acceptance or deliteralization repertoire in any context. FAP, FACT, and other interpersonally oriented psychotherapies bring this question to the forefront by focusing on the client's repertoires in-session. However, as a synthesis of treatments tied to a behavior analytic tradition, FACT is in a unique position to document this generalization.

One clear advantage of behavioral approaches to therapy remains the specification of problematic (and improved) response repertoires, including those that occur in the session. This specification allows measurement of the behaviors in order to document whether change has occurred. Devices such as the Functional Idiographic Assessment Template (FIAT; Callaghan et al., 2003) that idiograpically specify the client's problem behaviors in a FACT framework both in and out of session and then track those behaviors over time are currently being developed and tested. This measurement strategy provides the therapist and client with information about what is and is not working in treatment and what changes are occuring outside session.

\section{Conclusion and Future Directions}

This article has presented a brief summary of ACT and FAP, the strengths of each, the differ- ences between the two interventions, and the commonalities among them. We argue that the most comprehensive contemporary behavior analytic psychotherapy is a combination of the two, called FACT, where one therapy serves as the context for the other. This hybrid is hypothesized to be essential not only in producing improved clinical outcomes but also in the training of effective contemporary behavioral therapists.

One area of clinical investigation that needs to be addressed concerns whether there is an order of providing components of the interventions that is more useful for clients. Do clients benefit most from learning a basic acceptance repertoire prior to addressing interpersonal relationship problems, or is it essential to address acceptance in the context of a developing therapeutic relationship? This is not to suggest that FACT backtrack into a hierarchical arrangement of ACT before FAP or vice versa. We propose that these may be important empirical questions that would help the development of FACT as well as ACT and FAP as individual treatments. This article is the first in a series of proposals and studies to integrate two cutting-edge behavioral treatments. We invite other researchers to continue this line of investigation.

\section{References}

American Psychiatric Association. (2000). Diagnostic and statistical manual of mental disorders (4th ed., text rev.). Washington, DC: Author.

BACH, P., \& HAYES, S. C. (2002). The use of acceptance and commitment therapy to prevent the rehospitalization of psychotic patients: A randomized controlled trial. Journal of Consulting and Clinical Psychology, 70, 1129-1139.

Biglan, A., Glasgow, R. E., \& Singer, G. (1990). The need for a science of larger social units: A contextual approach. Behavior Therapy, 21, 195-215.

Blackledge, J. T., \& Hayes, S. C. (2001). Emotion regulation in acceptance commitment therapy. Journal of Clinical Psychology, 57, 243-255.

Callaghan, G. M., \& Gummeson, B. (2001, May). Examining interpersonal relationship skills using an idiographic assessment device. Poster session presented at the 81st Annual Meeting of the Western Psychological Association, Maui, HI.

Callaghan, G. M., Naugle, A. E., \& Follette, W. C. (1996). Useful constructions of the client-therapist relationship. Psychotherapy, 33, 381-390.

Callaghan, G. M., Summers, C. J., \& Weidman, M. (2003). The treatment of histrionic and narcissistic personality disorder behaviors: A single-subject demonstration of clinical effectiveness using functional analytic psychotherapy. Journal of Contemporary Psychotherapy, 33, 321-339.

Cinciripini, P. M., Hecht, S. S., Henningfield, J. E., ManLEY, M. W., \& Kramer, B. S. (1997). Tobacco addiction: 
Implications for treatment and cancer prevention. Journal of the National Cancer Institute, 89, 1852-1867.

Cordova, J. V., \& Kohlenberg, R. J. (1994). Acceptance and the therapeutic relationship. In S. C. Hayes, N. S. Jacobson, V. M. Follette, \& M. J. Dougher (Eds.), Acceptance and change: Content and context in psychotherapy (pp. 125-140). Reno, NV: Context Press.

Fiore, M. C., Bailey, W. C., Cohen, S. J., Dorfman, S. F., Goldstein, M. G., Gritz, E. R., ET AL. (1996). Smoking cessation, clinical practice guideline No. 18 (AHCPR Pub. No. 96-0692). Rockville, MD: U.S. Department of Health and Human Services, Public Health Service, Agency for Health Care Policy and Research.

Follette, W. C., Naugle, A. E., \& Callaghan, G. M. (1996). A radical behavioral understanding of the therapeutic relationship in effecting change. Behavior Therapy, 27, 623-641.

GAYNOR, S. T. (2002). Getting ahead of the data: Not all threats are equal. Behavior Therapist, 25, 137-139.

GAYNOR, S. T., \& LAWRENCE, P. S. (2002). Complementing CBT with learning through in vivo experience (LIVE): Conceptual analysis, treatment description, and feasibility study. Behavioural \& Cognitive Psychotherapy, 30, 79-101.

HawkINS, R. P. (1986). Selection of target behaviors. In R. O. Nelson \& S. C. Hayes (Eds.), Conceptual foundations of behavioral assessment (pp. 329-385). New York: Guilford Press.

HAYES, S. C. (1987). A contextual approach to therapeutic change. In N. Jacobson (Ed.), Psychotherapists in clinical practice: Cognitive and behavioral perspectives (pp. 327-387). New York: Guilford Press.

HAYES, S. C. (1994). Relational frame theory: A functional approach to verbal events. In S. C. Hayes \& L. J. Hayes (Eds.), Behavior analysis of language and cognition (pp. 9-29). Reno, NV: Context Press.

Hayes, S. C., Gifford, E. V., \& Wilson, K. G. (1996). Stimulus classes and stimulus relations: Arbitrarily applicable relational responding as an operant. In T. R. Zentall \& P. M. Smeets (Eds.), Stimulus class formation in humans and animals (pp. 279-299). New York: Elsevier.

Hayes, S. C., Strosahl, K., \& Wilson, K. G. (2000). Acceptance and commitment therapy: Treating human suffering. New York: Guilford Press.

Hayes, S. C., \& Wilson, K. G. (1994). Acceptance and commitment therapy: Altering the verbal support for experiential avoidance. The Behavior Analyst, 17, 289-303.

Hayes, S. C., Wilson, K. G., Bissett, R., Gifford, E. V., Piasecki, M., Batten, S. V., et Al. (2003, August). Acceptance and commitment therapy with polysubstance abusing methadone maintained opiate addicts: Outcome and process. Paper presented at the World Conference on ACT, RFT, and the New Behavioral Psychology, Linköping, Sweden.

Haynes, S. N., \& O'Brien, W. H. (2000). Principles and practice of behavioral assessment. New York: Kluwer/ Plenum.

KoHLENBERG, B. S., \& GifFord, E. (1998, May). FACT (FAP \& ACT): Clinical behavior analysts do it in-vivo. Paper presented at the 24th Annual Meeting of the Association for Behavior Analysis, Orlando, FL.

Kohlenderg, R. J., Hayes, S. C., \& Tsai, M. (1993). Radical behavioral psychotherapy: Two contemporary examples. Clinical Psychology Review, 13, 579-592.

Kohlenberg, R. J., Kanter, J. W., Bolling, M. Y., Parker, C. R., \& TsaI, M. (2002). Enhancing cognitive therapy for depression with functional analytic psychotherapy: Treatment guidelines and empirical findings. Cognitive and $\mathrm{Be}$ havioral Practice, 9, 213-229.

Kohlenberg, R. J., Kanter, J. W., Bolling, M., WeXner, R., Parker, C., \& Tsai, M. (2004). Functional analytic psychotherapy, cognitive therapy, and acceptance. In S. C. Hayes, V. M. Follette, \& M. Linehan (Eds.), The new behavior therapies: Expanding the cognitive behavioral tradition. New York: Guilford Press.

Kohlenberg, R. J., \& TsaI, M. (1991). Functional analytic psychotherapy: Creating intense and curative therapeutic relationships. New York: Plenum.

KoHLENBERG, R. J., \& TSAI, M. (1995). I speak therefore I am: A behavioral approach to understanding problems of the self. The Behavior Therapist, 18, 113-116.

LineHAN, M. M. (1993). Cognitive-behavioral treatment of borderline personality disorder. New York: Guilford.

Paul, R. H., MarX, B. P, \& Orsillo, S. M. (1999). Acceptance-based psychotherapy in the treatment of an adjudicated exhibitionist: A case example. Behavior Therapy, 30, 149-162.

PiASECKI, M. (2000). Smoking, nicotine, and mood. In M. Piasecki \& P. Newhouse (Eds.), Nicotine in psychiatry (pp. 139-148). Washington, DC: American Psychiatric Press.

Prins, A., \& Callaghan, G. M. (2002, November). Functional analytic psychotherapy as an adjunctive treatment for a client meeting criteria for PTSD. Poster session presented at the 36th Annual Meeting of the Association for the Advancement of Behavior Therapy, Reno, NV.

ROSENFARB, I. S. (1992). A behavior analytic interpretation of the therapeutic relationship. The Psychological Record, 42, 341-354.

SchaAp, C., Bennun, I., Schindler, L., \& Hoodguin, K. (1993). The therapeutic relationship in behavioural psychotherapy. New York: Wiley.

SwEET, A. A. (1984). The therapeutic relationship in behavior therapy. Clinical Psychology Review, 4, 253-272.

Zettle, R. D., \& Hayes, S. C. (1987). Component and process analysis of cognitive therapy. Psychological Reports, 61, 939-953.

Zettle, R. D., \& Rains, J. C. (1989). Group cognitive and contextual therapies in treatment of depression. Journal of Clinical Psychology, 45, 436-445. 